01

\title{
О силе электростатического взаимодействия между двумя проводящими сфероидами
}

\author{
(С) С.И. Гращенков
}

Псковский государственный университет, 180000 Псков, Россия

e-mail: grasi@mail.ru

Поступило в Редакцию 5 февраля 2019 г.

В окончательной редакции 3 декабря 2019 г.

Принято к публикации 15 декабря 2019 г.

Рассмотрено применение метода конечных элементов для расчета силы электростатического взаимодействия двух проводящих тел сфероидальной формы с заданными зарядами на их поверхностях без учета внешнего поля.

Ключевые слова: метод конечных элементов, электростатическое взаимодействие.

DOI: $10.21883 / J T F .2020 .05 .49169 .36-19$

\section{Введение}

Расчет сил взаимодействия двух заряженных проводящих тел важен для анализа различных физических процессов. Примером может служить расчет констант коагуляции в заряженных аэродисперсных системах. В том случае, когда расстояние между телами много больше их размеров, вычисление этих сил можно проводить на основе закона Кулона. Однако при близких расстояниях между телами существенную роль начинает играть поляризационное взаимодействие, что делает актуальным разработку методов, позволяющих проводить расчет сил электростатического взаимодействия проводящих тел при заданных величинах их зарядов. Проблема взаимодействия двух заряженных сферических проводящих тел разных радиусов впервые была рассмотрена С.Д. Пуассоном [1], в дальнейшем исследовалась У. Томсоном [2], Дж. Клерком Максвеллом [3] и рядом других исследователей (см., например, [4,5]), и ее окончательное решение была найдено лишь с появлением современной вычислительной техники [6,7]. Что же касается тел, форма которых отлична от сферической, то силы взаимодействия между ними вычислялись лишь при заданных значениях потенциалов на их поверхностях $[8,9]$, но не их зарядов. С практической же точки зрения представляет интерес и вычисление силы взаимодействия между проводящими телами с отличными от сферической формами при заданных зарядах на их поверхностях. В связи с этим в настоящей работе с помощью метода конечных элементов исследуется взаимодействие сфероидальных заряженных тел.

\section{Общая схема расчета}

Прежде чем перейти к вычислению сил электростатического взаимодействия двух проводников при заданных величинах их зарядов, рассмотрим способ расчета этих сил для известных значений потенциалов на поверхностях этих тел при условии, что вдали от них потенциал полагается равным нулю. Распределение потенциала $\varphi$ в окрестности проводников в этом случае однозначным образом находится из решения уравнения Лапласа [10]

$$
\Delta \varphi=0 .
$$

В методе конечных элементов область, в которой ищется распределение той или иной величины, разбивается на множество подобластей. В результате получается расчетная сетка, на основе которой генерируется набор базисных функций, используемых для аппроксимации искомого распределения. В настоящее время имеется ряд компьютерных программ, позволяющих находить численное решение исходной системы дифференциальных уравнений методом конечных элементов, а поиск распределения некоторой величины, являющейся решением уравнения (1) при заданных ее значениях на границах некоторой расчетной области, является, как правило, стандартным обучающим примером, на котором описываются особенности использования той или иной программы в ее руководстве. Примерами таких программ являются freefem ${ }^{++}$[11] и FEniCS [12]. Так же именно этот пример обычно первым рассматривается в различных руководствах по методу конечных элементов. Ввиду большого количества литературы по данному вопросу, в настоящей статье подробности применения этого метода к решению уравнения (1) опускаются. В методе конечных элементов распределение той или иной величины всегда ищется в некоторой конечной области. Очевидно, что в нашем случае размер этой области должен быть велик по сравнению с размерами рассматриваемых тел и расстоянию между ними для того, чтобы соблюсти условие равенства нулю потенциала на большом расстоянии от тел. Таким образом, задавая нужные значения потенциала на поверхностях проводников и нулевое значение на удаленных от них границах и используя ту или иную программу, мы можем 
легко найти распределение потенциала в окрестностях тел. Далее по найденному распределению можно найти распределение напряженности $E$ электрического поля, а по распределению напряженностей вблизи поверхности тела найти действующую на тело силу при помощи выражения [13]

$$
F=-\frac{\varepsilon_{0}}{2} \oint_{S} E^{2} \mathbf{n} d S
$$

Здесь $\mathbf{n}$ - единичный вектор нормали к элементу поверхности, направленный внутрь тела, $S$ - площадь поверхности тела, $\varepsilon_{0}$ - электрическая постоянная.

Для того чтобы от рассмотренной задачи перейти к задаче о вычислении силы взаимодействия проводящих тел при их известных зарядах, воспользуемся известной формулой $[3,10]$, связывающей эту силу $F$ и заряды $Q_{1}$ и $Q_{2}$ соответственно первого и второго тела:

$$
F=k_{11} Q_{1}^{2}+k_{12} Q_{1} Q_{2}+k_{22} Q_{2}^{2} .
$$

Здесь $k_{11}, k_{12}, k_{22}$ - коэффициенты, значения которых зависят от размеров и формы тел и расстояния между ними. При расчетах будем полагать, что отталкиванию тел в этой формуле соответствует положительное значение $F$, а притяжению - отрицательное. Таким образом, задача о нахождении силы между телами при заданных зарядах сводится к задаче о нахождении коэффициентов $k_{11}, k_{12}, k_{22}$. Для нахождения этих коэффициентов достаточно три раза выбрать некоторые значения потенциалов $\varphi_{1}$ и $\varphi_{2}$, и в каждом случае по формуле (2) определить силу взаимодействия между телами, а по теореме Гаусса - заряды этих тел. Подставляя полученные значения величин в формулу (3), получим три уравнения для расчета трех неизвестных коэффициентов $k_{11}, k_{12}, k_{22}$. Соответствующие тестовые расчеты для шаров равных радиусов, проведенные при помощи программы freefem ${ }^{++}$(см. ниже) и при помощи программы FEniCS, и последующее сравнение полученных значений коэффициентов со значениями, полученными на основе аналитических выражений [7], показали, что такой подход действительно применим, если кратчайшее расстояние $h$ между поверхностями тел не превышает трех радиусов тел, т. е. сравнимо с их размерами. Дальнейшее увеличение расстояния приводит к увеличению погрешности расчета коэффициентов $k_{11}$, $k_{22}$, которая при радиусе тел $R$ и расстоянию между их поверхностями $h=10 R$ может достигать 50\%. Отметим, что уже при $h=3 R$ значение коэффициентов $k_{11}, k_{22}$ не превышают $2 \%$ от $k_{12}$, поэтому в случае сравнимых по величине зарядов $Q_{1}$ и $Q_{2}$ рассмотренный подход вполне применим, так как при $h>3 R$ в этой ситуации становится возможным использование закона Кулона. Однако при значительно отличающихся величинах этих зарядов или при исследовании поляризационного взаимодействия заряженного тела с незаряженным желательно обеспечить большую точность вычислений. Следует также отметить, что точность вычисления рассматриваемых коэффициентов существенным образом зависела от того, какое тело при составлении соответствующего уравнения выбиралось для вычисления силы взаимодействия. Поясним сказанное. Хотя по третьему закону Ньютона модули сил, действующих на тела и вычисляемых по формуле (2), должны быть равны, вследствие погрешностей вычисления их значения получаются разными, и в случае сильно различающихся получаемых по теореме Гаусса зарядов тел погрешность расчета силы, действующей на ту частицу, заряд по модулю которой получается большим, оказывается большей. Поэтому простое использование для расчета сил взаимодействия тела с меньшим зарядом при составлении соответствующего уравнения рассматриваемой выше системы приводит к значительному уменьшению погрешности вычислений коэффициентов, входящих в уравнение (3). Рассмотрим причину этого обстоятельства. Поскольку метод конечных элементов по своей сути является приближенным, вследствие ошибок вычисления получаемое распределение потенциала флуктуирует около некоторого истинного распределения. При вычислении поверхностных интегралов от функций, содержащих эти распределения, влияние этих флуктуаций накапливается, и значения таких интегралов могут вычисляться со значительной погрешностью. В нашем случае это приводит к заметному понижению точности расчета сил по формуле (2), когда изменение напряженности вдоль поверхности тела мало по сравнению с ее средним значением. В связи с этим для расчета коэффициентов, входящих в формулу (3), рассмотренный выше алгоритм вычислений был модифицирован так, чтобы при расчете коэффициентов в двух уравнениях системы использовались силы, рассчитанные по поверхности тела с зарядом, равным нулю, а для составления третьего уравнения использовались равные заряды. Для этого применялись выражения, связывающие заряды тел и потенциалы их поверхностей [10]:

$$
\begin{aligned}
& Q_{1} p_{11}+Q_{2} p_{21}=\varphi_{1}, \\
& Q_{2} p_{12}+Q_{2} p_{22}=\varphi_{2},
\end{aligned}
$$

где $p_{11}, p_{21}, p_{12}, p_{22}$ - потенциальные коэффициенты. В итоге получился следующий алгоритм.

1. Задаем на поверхности первого тела отличный от нуля потенциал $\varphi_{1}$. На бесконечности и поверхности второго тела потенциал полагаем равным нулю. Считаем по теореме Гаусса заряды первого и второго тел. Используя выражения (4), получаем первые два уравнения для расчета потенциальных коэффициентов. Меняем местами потенциалы тел и получаем еще два уравнения. Решая полученную систему, находим потенциальные коэффициенты.

2. Выбираем некоторый ненулевой заряд первого тела, а заряд второго тела полагаем равным нулю. По формулам (4) вычисляем соответствующие потенциалы тел. Используя эти потенциалы, находим решение уравнения (1). По формуле (2) рассчитываем силу, действующую на второе тело, для которого точность 
вычислений сил в данном случае максимальна. Вновь по теореме Гаусса вычисляем заряды тел. Вследствие погрешностей расчета эти заряды будут несколько отличаться от исходных, и даже при $Q_{1} \gg Q_{2}$, в ситуациях, когда $k_{11} \ll k_{12}$, значения слагаемых $k_{11} Q_{1}^{2}$ и $k_{12} Q_{1} Q_{2}$ могут оказаться сравнимыми друг с другом. Подставляя полученные значения силы и зарядов в формулу (3), получаем первое уравнение для расчета входящих в нее коэффициентов.

3. Меняем местами заряды тел и, повторяя описанную в п. 2 процедуру, но с расчетом силы, действующей на первое тело, получаем второе уравнение для расчета входящих в формулу (3) коэффициентов.

4. Суперпозиция полученных в п. 2 и 3 распределений потенциала дает, как следует из уравнения (1), решение для случая взаимодействия тел, заряды которых равны суммам соответствующих зарядов, полученных в этих пунктах. При этом, как показали тестовые расчеты, немного улучшить точность вычислений можно, если при вычислении сил использовать непосредственно распределения напряженностей, полученные в предыдущих пунктах, а не их суперпозицию. Поясним сказанное. Пусть мы вычисляем силу, действующую на второе тело, $\mathbf{E}_{1}$ - распределение напряженностей, полученное в п. 2, а $\mathbf{E}_{2}$ - распределение напряженностей, полученное в п. 3. Итоговое распределение $\mathbf{E}=\mathbf{E}_{1}+\mathbf{E}_{2}$. Подставляя это выражение в (2), получаем

$$
\mathbf{F}=-\frac{\varepsilon_{0}}{2} \oint_{S} E_{1}^{2} \mathbf{n} d S-2 \varepsilon_{0} \oint_{S} \mathbf{E}_{1} \mathbf{E}_{2} \mathbf{n} d S-\frac{\varepsilon_{0}}{2} \oint_{S} E_{2}^{2} \mathbf{n} d S .
$$

Но первое слагаемое дает силу, рассчитанную в п. 2, а третье должно совпадать с силой, рассчитанной в п. 3, и именно значения этих сил мы будем использовать вместо этих слагаемых. В результате вместо третьего слагаемого мы будем использовать более точное значение, чем в расчете по итоговой суперпозиции напряженностей.

5. Из полученной системы уравнений находим значение коэффициентов $k_{11}, k_{12}, k_{22}$.

Тестовые расчеты значений этих коэффициентов с использованием изложенного алгоритма для шаров, проведенные при помощи программы freefem ${ }^{++}$, и последующее их сравнение со значениями, полученными на основе аналитических выражений [7], показали, что данный алгоритм при $h=10 R$ позволяет вычислять указанные коэффициенты для одинаковых шаров с погрешностями менее полупроцента, а для двух шаров с радиусами, отличающимися в два раза (в этом случае $R-$ радиус большего шара), - c погрешностями, не превышающими $0.6 \%$. Отметим, что все дальнейшие расчеты по данному алгоритму проводились именно при помощи данной программы, а программа FEniCS использовалась лишь для получения дополнительного подтверждения того, что большие погрешности расчета на основе первоначально рассмотренного подхода обусловлены именно недостатками метода конечных элементов, а не его реализацией в программе freefem ${ }^{++}$.

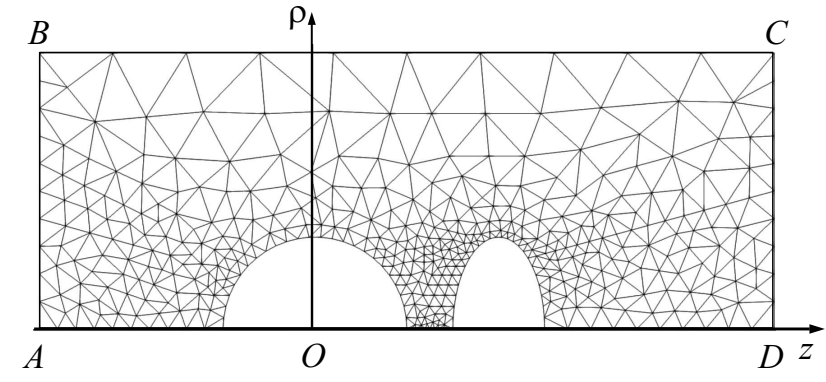

Рис. 1. Структура исходной расчетной области.

\section{Исследование влияния поляризационного взаимодействия}

Разработанный алгоритм расчета был применен для исследования поляризационного взаимодействия частиц сфероидальной формы. Как уже было сказано, для расчетов использовалась программа freefem ${ }^{++}$. Поскольку в методе конечных элементов расчет всегда ведется в конечной области, границы этой области полагались достаточно удаленными от тел, и задание нулевого потенциала на бесконечности моделировалось его заданием на этой границе. Если искомые распределения осесимметричны, как это имеет место в данном случае, требования к вычислительным ресурсам можно многократно сократить, перейдя к цилиндрическим координатам, и в результате трехмерную задачу свести к двумерной, в которой все распределения зависят только от полярного радиуса $\rho$ и аппликаты $z$ цилиндрической системы координат. Сведения, необходимые для перехода к цилиндрической системе координат, приведены в [14] и в руководстве к программе freefem ${ }^{++}$. Рассмотрим построение расчетной сетки. Пусть для определенности первое тело имеет форму шара, а вторая частица сплюснутого сфероида. Структура расчетной области для этого случая показана на рис. 1. Для удобства отображения отдельных элементов соотношения их размеров отличаются от реально используемых. Обозначим через $а$ расстояние от центра второй частицы до ее поверхности вдоль оси $z$, а через $b-$ расстояние от центра этой частицы до ее поверхности в направлении, перпендикулярном этой оси. Последнее расстояние в данном случае равно радиусу $R$ первого тела. При построении расчетной сетки расстояние $R$ (или $b$ в случае двух одинаковых сплюснутых сфероидов) принималось равным единице. Расстояния от центра первого тела частицы до левой границы расчетной области, от центра второй частицы до правой границы и от оси $z$ до верхней границы полагались равными $50 R$ (или $50 \mathrm{~b}$ в случае двух одинаковых сплюснутых сфероидов). Как показали результаты расчетов, для одинаковых шаров дальнейшее увеличение размеров указанной области не приводило к заметному изменению получаемых результатов. Если шар рассматривать, как частный случай сфероида, то все далее используемые формы тел получались только 


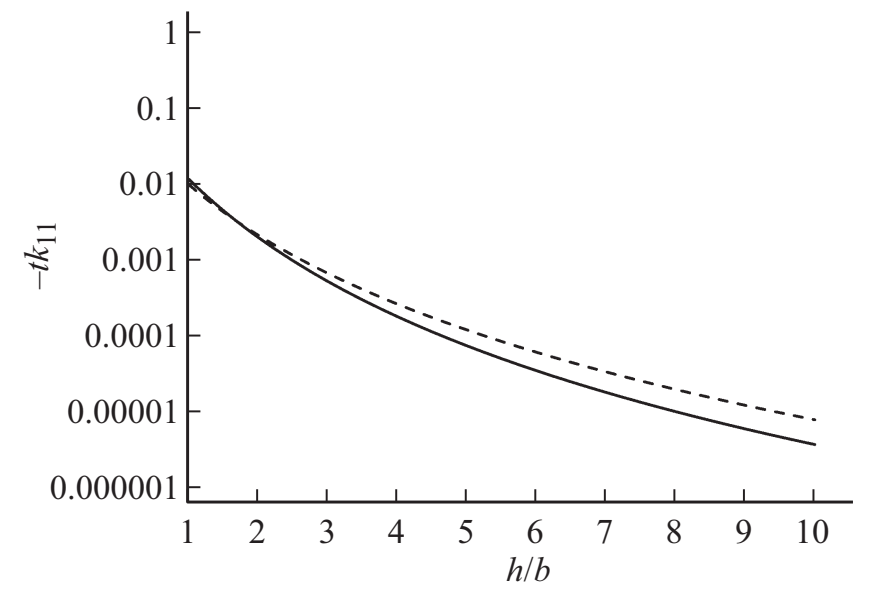

Pис. 2. Зависимости нормированного значения коэффициента $k_{11}$ от относительного расстояния между поверхностями тел для двух сплюснутых сфероидов с полуосями $b$ и $a$ (сплошная линия) и шаров (штриховая линия) с радиусами $R, R=b=2 a$.

уменьшением той или иной полуоси этих тестовых сфероидов, что гарантировало достаточность размеров области для корректности расчета в этих случаях. Разбиение рассматриваемой области на подобласти производилось при помощи алгоритма Делоне-Вороного [15]. Программа freefem ${ }^{++}$проводит такое разбиение автоматически по заданному числу точек на границах. Число подобластей расчетной области в зависимости от форм тел и расстояний между ними менялось от $2 \cdot 10^{4}$ до $8 \cdot 10^{4}$. Для аппроксимации искомого распределения потенциалов использовались базисные функции на основе полиномов Лежандра третьего порядка. Время одного расчета с использованием процессора Intel $\mathbb{B}$ Core $\mathbb{R}$ i3-210 не превышало полуминуты. Потенциал на границе ABCD расчетной области полагался равным нулю. На границах расчетной области, соответствующих поверхностям тел, задавались их потенциалы. На границе AOD, совпадающей с осью симметрии, никаких условий в явном виде не задавалось, так как использование цилиндрической системы координат при нахождении методом конечных элементов решения уравнения Лапласа фактически вводит неявное граничное условие равенства нулю нормальной составляющей градиента вычисляемой величины на границе, совпадающей с осью $z$ [14].

На рис. 2 сплошной линией для двух одинаковых тел, имеющих форму сплюснутого сфероида с $b=2 a$, изображена зависимость величины $-t k_{11}$ от кратчайшего расстояния $h$ между поверхностями тел, где нормировочный множитель $t$ имеет вид

$$
t=4 \pi b^{2} \varepsilon_{0} .
$$

Штриховой линией для сравнения изображена зависимость этой же величины для двух одинаковых шаров радиусом $R=b$. Так как тела одинаковы, то $k_{22}=k_{11}$. Обратим внимание на то, что величина $-t k_{11}$ представляет собой силу притяжения между телами, нормированную на $Q_{1}^{2} / t$, для случая, когда первое тело имеет ненулевой заряд $Q_{1}$, а заряд второго тела равен нулю. Из рисунка видно, что на больших расстояниях эта сила для сплюснутых сфероидов меньше, чем для шаров, а на малых - немного больше. Вместе с тем, как видно из рис. 3 , величина $k_{12}$, характеризующая вклад взаимного отталкивания помещенных на тела одноименных зарядов в величину итоговой силы, при всех рассмотренных расстояниях больше для сплюснутого сфероида, чем для шара. В итоге для сплюснутых тел интервал отношения $Q_{1} / Q_{2}$ одноименных зарядов тел, при котором они отталкиваются, оказывается больше, чем для шаров, что видно из рис. 4, где приведены зависимости этого отношения от расстояния между поверхностями тел, при котором сила взаимодействия между телами равна нулю. Отметим, что если рассматривать зависимости коэффициентов $k_{11}, k_{12}, k_{22}$ от расстояния $r$ между центрами тел, то при всех $r$ модули этих величин окажутся больше для шаров. Однако значения суммы

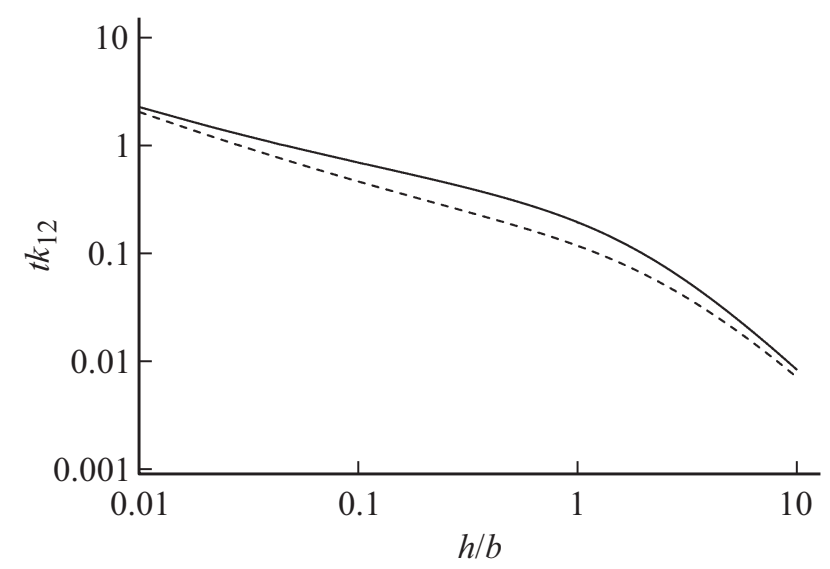

Рис. 3. Зависимости нормированного значения коэффициента $k_{12}$ от относительного расстояния между поверхностями тел для двух сплюснутых сфероидов с полуосями $b$ и $a$ (сплошная линия) и шаров (штриховая линия) с радиусами $R, R=b=2 a$.

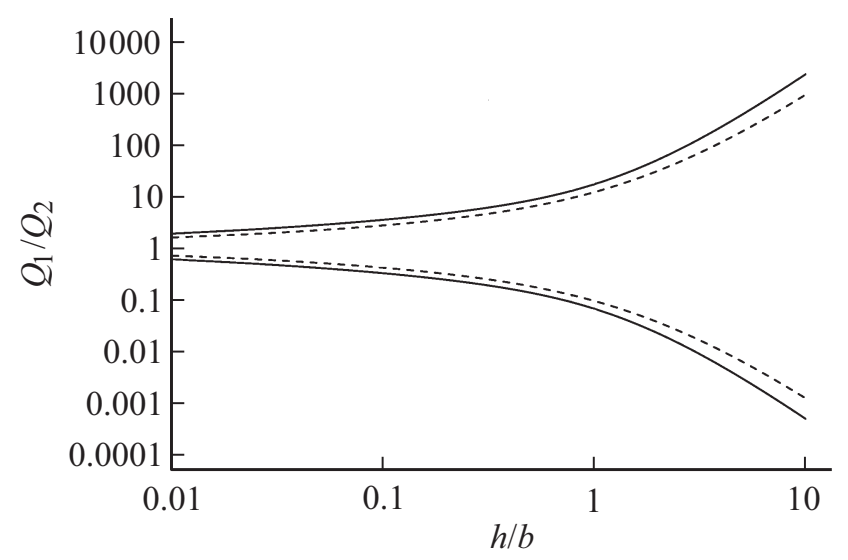

Рис. 4. Зависимости отношения зарядов тел от относительного расстояния между их поверхностями, при котором сила взаимодействия равна нулю, для двух сплюснутых сфероидов с полуосями $b$ и $a$ (сплошная линия) и шаров (штриховая линия) с радиусами $R, R=b=2 a$. 


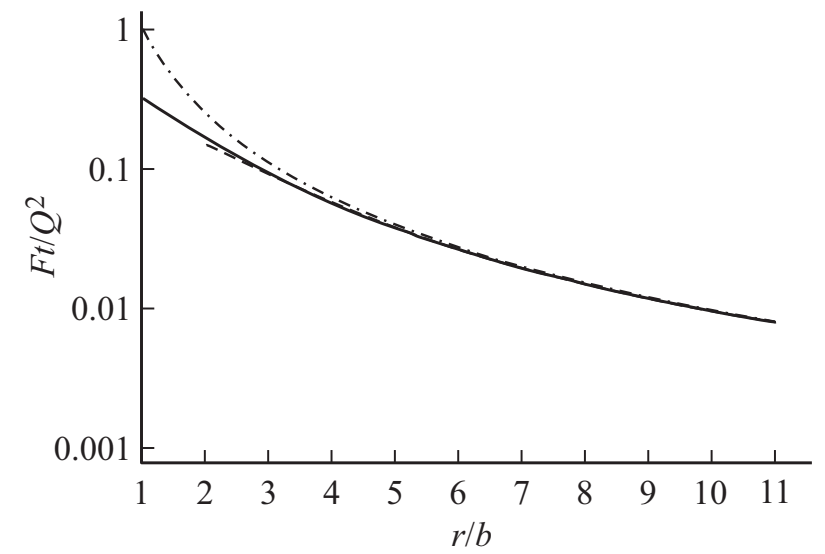

Рис. 5. Зависимости нормированной силы отталкивания между телами от относительного расстояния между центрами тел для двух сплюснутых сфероидов с полуосями $b$ и $a$ (сплошная линия), шаров с радиусами $R$ (штриховая линия) и точечных зарядов (штрихпунктирная линия) при одинаковых зарядах тел $Q_{1}=Q_{2}=Q, R=b=2 a$.

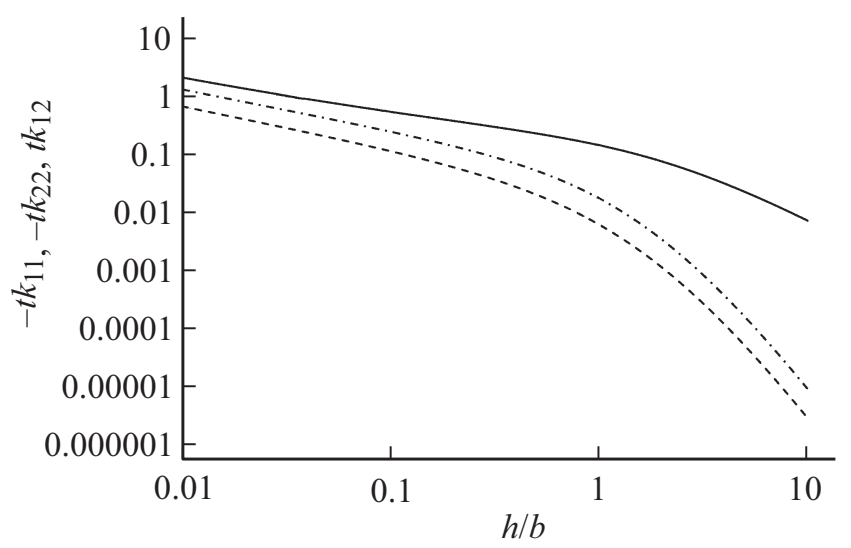

Рис. 6. Зависимости нормированных значений коэффициентов $k_{12}$ (сплошная линия), $k_{11}$ (штриховая линия), $k_{22}$ (штрихпунктирная линия) от относительного расстояния между поверхностями тел для шара с радиусом $R$ и сплюснутого сфероида с полуосями $b$ и $a, R=b=2 a$.

этих коэффициентов для шаров и сплюснутых сфероидов при одинаковых $r$ оказываются достаточно близки. Но эта сумма представляет собой нормированную на $Q^{2}$ силу отталкивания двух тел, заряд каждого их которых $Q_{1}=Q_{2}=Q$. Сказанное иллюстрируется рис. 5. Штрихпунктирной линией на этом рисунке для сравнения показана зависимость от $r$ величины $F t / Q^{2}$ для точечных зарядов.

Рассмотрим теперь взаимодействие шара со сплюснутым сфероидом при $R=b=2 a$. На рис. 6 приведены зависимости нормированных значений коэффициентов $k_{11}, k_{12}, k_{22}$ от расстояния между поверхностями тел. Из рисунка видно, что при всех расстояниях значение модуля коэффициента $k_{22}$ больше модуля коэффициента $k_{11}$. Расчеты показывают, что это верно и том случае, когда второе тело является вытянутым сфероидом при
$R=2 b=a$ или шаром с радиусом, в два раза меньшим радиуса первого шара. Это означает, что во всех случаях помещение некоторого заряда на одно из тел приводит к силе взаимного притяжения тел, которая оказывается большей, когда заряд помещается на второе тело. Эти результаты согласуются с результатами работы [5] для шара и точечного заряда. Кроме того, во всех рассмотренных случаях значение модуля коэффициента $k_{11}$ оказывается меньшим, чем для одинаковых шаров радиуса $R$, а значение модуля коэффициента $k_{22}$ - большим. Таким образом, если рассматривать два одинаковых тела шарообразной формы, одно из которых заряжено, то уменьшение размера незаряженного тела как вдоль оси $z$, так и поперек этой оси приводит к уменьшению сил притяжения между телами. Уменьшение же размеров заряженного тела при данном расстоянии между поверхностями тел приводит к увеличению этой силы. На рис. 7 для случая взаимодействия шара со сплюснутым сфероидом при $R=b=2 a$ приведены зависимости

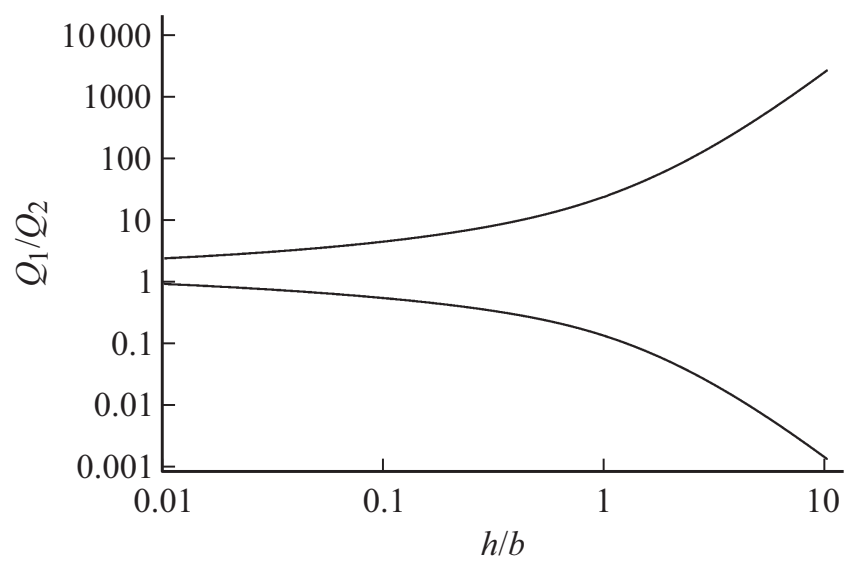

Рис. 7. Зависимости отношения зарядов тел от относительного расстояния между их поверхностями, при котором сила взаимодействия равна нулю, для шара с радиусом $R$ и сплюснутого сфероида с полуосями $b$ и $a, R=b=2 a$.

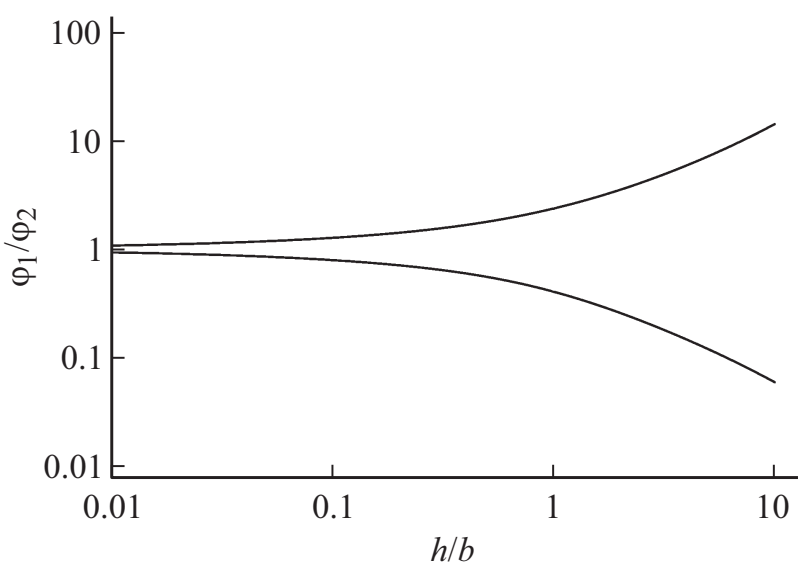

Рис. 8. Зависимости отношения потенциалов тел от относительного расстояния между поверхностями, при котором сила взаимодействия равна нулю, для шара с радиусом $R$ и сплюснутого сфероида с полуосями $b$ и $a, R=b=2 a$. 
отношения $Q_{1} / Q_{2}$ от расстояния между поверхностями тел, при котором сила взаимодействия между телами равна нулю, а на рис. 8 - отношения потенциалов этих тел при указанных отношениях зарядов. Из этих рисунков видно, что отношения зарядов при $Q_{1}>Q_{2}$ и при $Q_{1}<Q_{2}$ стремятся друг к другу, а отношения потенциалов - к единице. Из того следует, что, как и в случае шаров [7], одноименно заряженные тела отталкиваются друг от друга при любых расстояниях между ними только при условии равенства отношения их зарядов отношению, которое получается в результате перераспределения общего заряда тел при их контакте. Аналогичные выводы вытекают и из расчетов для шара и вытянутого сфероида.

\section{Заключение}

С использованием метода конечных элементов рассмотрено электростатическое взаимодействие заряженных проводников в виде двух сфероидов. На примерах взаимодействия шаров и взаимодействия сплюснутых сфероидов показано, что при заданном расстоянии между поверхностями одинаковых тел уменьшение их продольного размера (т. е. размера вдоль соединяющей их линии) увеличивает интервал отношения зарядов этих тел, при котором они отталкиваются. Расчеты сил взаимодействия шара и сплюснутого или вытянутого сфероида показали, что при заданном расстоянии между поверхностями первоначально одинаковых тел, только одно из которых заряжено, сила притяжения между ними уменьшается при уменьшении как поперечного, так и продольного размера незаряженного тела и увеличивается, если уменьшаются размеры заряженного тела, а одноименно заряженные тела отталкиваются друг от друга при любых расстояниях между ними только при условии равенства отношения их зарядов отношению, которое получается в результате перераспределения общего заряда тел при их контакте.

\section{Конфликт интересов}

Автор заявляет, что у него нет конфликта интересов.

\section{Список литературы}

[1] Уиттекер Э. История теории эфира и электричества. Классические теории. Ижевск: НИЦ „Регулярная и хаотическая динамика“, 2001. 512 с. [Whittaker E. A history of the theories of aether and electricity. The classical theories. NY.: Thomas Nelson and Sons, 1951. 434 p.]

[2] Thomson $W$. On the mutual attraction or repulsion between two electrified spherical conductors. In Reprint of Papers on Electrostatics and Magnetism (Cambridge Library Collection-Physical Sciences, P. 86-97). Cambridge: Cambridge University Press. 2011.

DOI: $10.1017 /$ cbo9780511997259.007
[3] Максвелл Джс.К. Трактат об электричестве и магнетизме. М.: Наука, 1989. [Maxwell J.C. A Treatise on Electricity and Magnetism, Vol. 1 (3rd Edition). NY.: Dover Publications Inc., 1954. 560 p.]

[4] Щерба Е.А., Коромыслов В.А., Григорьев А.И. // ЖТФ. 2002. Т. 72. Вып. 1. С. 15-19. [Shcherba E.A., Grigor'ev A.I., Koromyslov V.A. // Tech. Phys. 2002. Vol. 47. N 1. P. 13-17. DOI: https://doi.org/10.1134/1.1435884]

[5] Григорьев А.И. // ЖТФ. 2010. Т. 80. Вып. 5. С. 75-79. [Grigor'ev A.I. // Tech. Phys. 2010. Vol. 55. N 5. P. 666-671. DOI: https://doi.org/10.1134/S1063784210050105]

[6] Davis M.H. // Quart. J. Mech. Appl. Math. 1964. Vol. 17. N 4. P. 499-511. DOI: https://doi.org/10.1093/qjmam/17.4.499

[7] Гращенков С.И. // ЖТФ. 2011. Т. 81. Вып. 7. С. 13-17. [Grashchenkov S.I. // Tech. Phys. 2011. Vol. 56. N 7. P. 914 918. DOI: https://doi.org/10.1134/S1063784211070115]

[8] Murovec T., Brosseau C. // Appl. Phys. Lett. 2013. Vol. 102. N 8. P. 084105-1-084105-5. DOI: http://dx.doi.org/10.1063/1.4793664

[9] Khair A.S. // J. Appl. Phys. 2013. Vol. 114. N 13. P. 1349061-134906-6. DOI: http://dx.doi.org/10.1063/1.4824540

[10] Смайт В. Электростатика и электродинамика. М.: ИЛ, 1954. 606 c. [Smythe W.B. Static and dynamic electricity. NY.: Hemisphere Publishing, 1988. 626 p.]

[11] Hecht F. // J. Numerical Mathem. 2012. Vol. 20. N 3-4. P. 251.

[12] Logg A., Mardal K.-A., Wells G.N. et al. Automated Solution of Differential Equations by the Finite Element Method. Berlin:Springer, 2012. $723 \mathrm{p}$. DOI: $10.1007 / 978-3-642-23099-8$

[13] Иродов И.Е. Основные законы электромагнетизма. Учеб. пособие для студентов вузов. 2-е, стереотип. М.: Высш. шк., 1991. 289 с.

[14] Reddy J.N., Gartling D.K. The Finite Element Method in Heat Transfer and Fluid Dynamics. Boca Raton: CRC Press, 2010. $489 \mathrm{p}$.

[15] Lucquin B., Pironneau $O$. Introduction to Scientific Computing. Chichester: Wiley, 1998. 361 p. 